\title{
Optimization of parameters and operating modes of the rotary working body for harrowing agricultural crops
}

\author{
Alexander Serguntsov ${ }^{1, *}$ and Victoria Serguntsova ${ }^{2}$ \\ ${ }^{1}$ Chair of processes and machines in agribusiness, Kuban State Agrarian University named after I. T. \\ Trubilin, 350044 Krasnodar, Russia \\ ${ }^{2}$ Department of law, 385000 Adygea State University, Maikop, Russia
}

\begin{abstract}
The rotary working body with evenly spaced needles along the perimeter is a flat disk (rotating in a longitudinally vertical plane). The technological process of interaction with the soil consists in the fact that when the rotary disk is rolled in the soil layer, the needle is inserted into it, crushing and shifting the soil in the direction of rotation. The degree of soil loosening, rotating freely in a longitudinal vertical plane of the needle disk on the axis depends on several parameters: disk diameter, number of needles, speed, and soil properties. An experimental study of needle working bodies was performed in terms of the influence of the disk size, the number of needles and the speed of movement on the process of soil crumbling.
\end{abstract}

\section{Materials and methods of the research}

Harrowing is a very important way to take care of crops. It is used to destroy the soil crust, especially on heavy flooding soils. As a rule, heavy rains that fall during the period from sowing to the emergence of seedlings, followed by hot dry weather, lead to the formation of a soil crust. It prevents air access to seeds and makes it difficult for plants to germinate. As a result, the seedlings are greatly thinned, partially die, and as a result, give non-uniform shoots [5]. All this reduces the yield and quality of agricultural products greatly. The needle harrow is used in the cultivation of winter wheat, its use should preserve moisture reserves in the soil, promote soil aeration. Using such unit allows us to maintain the water balance due to the interruption of capillarity to the surface - sealing of moisture (dry watering). The technological process of a multi-functional unit that combines several operations performed in a single pass through the field implies the presence of an appropriate set of agricultural machines in the design [2]. This unit must be equipped with working bodies for loosening the soil to a specified depth and a machine for applying fertilizers. In this regard, it is an urgent task to study the parameters and operating modes of the rotary working body for harrowing crops [3].

\footnotetext{
* Corresponding author: sasha2008_9191@mail.ru
} 


\section{Results of the research}

When using mathematical processing of the experiment data according to the $B_{k}$ plan, it allowed us to obtain the equation of soil crumbling by a needle-like working body with imaginary coefficients depending on the factors that we selected and related to the actual presented dependencies:

$$
x_{1}=\frac{X_{1}-X_{10}}{\Delta_{1}}, x_{2}=\frac{X_{2}-X_{20}}{\Delta_{2}}, x_{3}=\frac{X_{3}-X_{30}}{\Delta_{3}}
$$

where $x_{1}, x_{2}, x_{3}$ - values of imaginary factors;

$X_{1}, X_{2}, X_{3}$ - values of real factors;

$X_{10}, X_{20}, X_{30}$ - values of real factors in the center of the plan;

$\Delta_{1}, \Delta_{2}, \Delta_{3}$ - interval of varying factors.

The equation of crumbling of the surface soil layer by a rotary working body depending on the selected factors will take the form:

$$
\begin{aligned}
& Y=85,0153+1,851 \cdot x_{1}+2,503 \cdot x_{2}-1,066 \cdot x_{3}+1,2375 \cdot x_{1} \cdot x_{2}+ \\
& +0 \cdot x_{1} \cdot x_{3}+0,105 \cdot x_{2} \cdot x_{3}+1,038 \cdot x_{1}^{2}+2,768 \cdot x_{2}^{2}+2,753 \cdot x_{3}^{2}
\end{aligned}
$$

where $Y$ - soil crumbling with a rotation disk.

Using the Student's criterion, the coefficients of the regression equation (2) were checked for confidence intervals. The table value of the Student's criterion $t=2.0423$ with the number of degrees of freedom equal to 9 and 5\% significance level [1]. The coefficients of the regression equation according to the confidence intervals will be equal:

$$
\Delta b_{0}=1784,3 ; \Delta b_{i}=38,8532 ; \Delta b_{i j}=25,976 ; \Delta b_{i i}=22,3758
$$

where $\Delta b$ - confidence interval.

Using the Fischer criterion, we checked whether the resulting model was adequate, where the table value was 3.7 and the calculated value was 2.99, which confirms the adequacy of the model. Differentiating the regression equation (2) for each factor, we solved a system of linear equations to find the optimal values of factors. The imaginary values of the factors are equal to: $x_{1}=-0,715, x_{2}=-0,296, x_{3}=0,199$.

By substituting the optimal values of factors in the regression equation (2), we obtained an optimization in the center of the response surface, while $Y_{s}=83,877 \%$ [1].

The studied factors in the natural value [1]: the optimal speed of movement $V_{\mathrm{p}}=6.43$ $\mathrm{km} / \mathrm{h}$; the optimal diameter of the disk $D=518 \mathrm{~mm}$; the optimal number of needles (gears) $\mathrm{n}=14$ PCs.

We performed a canonical transformation for the obtained mathematical model of the regression equation in connection with the study of the properties of the response surface in the vicinity of the optimum. The origin of the coordinates of the mathematical model is moved to a new point, and the linear terms disappear from the equation, as well as the pair interactions disappear when turning at a certain angle, and the value of the free term also changes [1].

The angle of rotation $\alpha$ in our case was $40.935^{\circ}$. The regression equation (2) in canonical form will take the form:

$$
Y-83,877=1,038 \cdot X_{1}^{2}+2,768 \cdot X_{2}^{2}+2,753 \cdot X_{3}^{2}
$$

or: 


$$
\frac{\left(X_{1}^{2}\right)}{\frac{Y-83,877}{1,038}}+\frac{\left(X_{2}^{2}\right)}{\frac{Y-83,877}{2,768}}+\frac{\left(X_{3}^{2}\right)}{\frac{Y-83,877}{2,753}}
$$

where $Y$ - the number of the optimization criterion (cracking soil), $\mathrm{N}$;

$X_{1}, X_{2}, X_{3}$ - new coordinate axes turned in space, to an angle $\alpha$ relative to the old axes $\left(x_{1}, x_{2}, x_{3}\right)$

Using two-dimensional sections, the response surface was studied for a more detailed representation. When considering changes in the value of the optimization criterion depending on the values of the other two factors, we substituted the imaginary value of one of the optimal parameters in the original regression equation (2).

Using two-dimensional sections, the response surface was studied for a more detailed representation. When considering changes in the value of the optimization criterion depending on the values of the other two factors, we substituted the imaginary value of one of the optimal parameters in the original regression equation $x_{1}=-0,715$ (2):

$$
Y_{23}=84,23+1,623 \cdot x_{2}-1,066 \cdot x_{3}+0,105 \cdot x_{2} \cdot x_{3}+2,768 \cdot x_{2}^{2}+2,753 \cdot x_{3}^{2}
$$

where $Y_{23}$-function of soil crumbling by a rotary working body, g.

After the canonical transformation, the equation (5) with the angle of rotation $\alpha$ equal to $0^{\circ}$, it will take the form (6), and the coefficients of this equation will be equal to: $B_{22}=2,814, B_{33}=2,707$. [1]:

$$
Y_{23}-83,877=2,814 \cdot X_{2}^{2}+2,707 \cdot X_{3}^{2}
$$

The surface represented (in Fig. 1) and the equation obtained by the regression (6) is an elliptical paraboloid. The center of the surface is the minimum, since the coefficients of the equation are positive. Due to the fact that the ellipse, along the axis that corresponds to the number of needles, is elongated more in this two-dimensional section, we conclude that the diameter of the working body affects the response value more than the number of gears (needles).
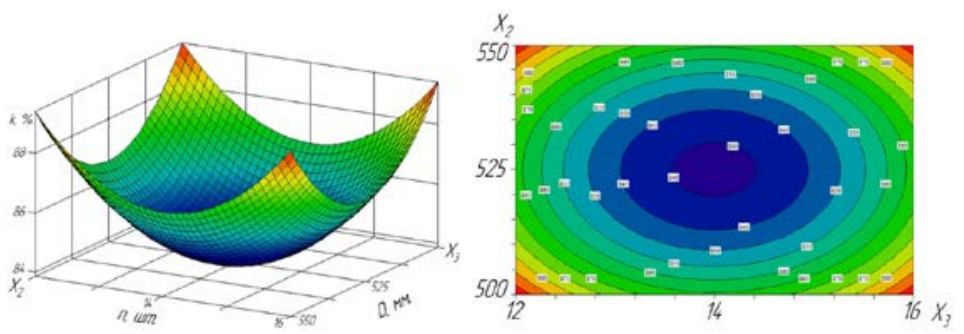

Fig. 1. The surface of the dependence of soil crumbling on the diameter of the disk and the number of needles (gears) and its two-dimensional cross-section

Similarly, let's consider how the number of needles and the speed of movement affect the change in the response value for an optimal value of the disk diameter. To do this, it is necessary to substitute the optimal imaginary value of the disk diameter in the regression equation $x_{2}=-0$, 296. (2)

Then the equation takes the form:

$$
Y_{13}=84,51+1,485 \cdot x_{1}-1,097 \cdot x_{3}+1,038 \cdot x_{1}^{2}+2,753 \cdot x_{3}^{2}
$$


After the canonical transformation, equation (7) with the angle of rotation $\alpha$ equal to $0^{\circ}$, it will take the form (8), and the coefficients of this equation will be equal to: $B_{11}=1,038$, $B_{33}=2,753$. The surface shown in (Fig. 2) and obtained from the regression equation (8) is an elliptical paraboloid. The center of the surface is the minimum, since the coefficients of the equation are positive.

Equation (8) in canonical form has the form:

$$
Y_{13}-83,877=1,038 \cdot X_{1}^{2}+2,753 \cdot X_{3}^{2}
$$

According to the cross-section obtained (Fig. 2), the response value is more influenced by the change in the number of needles (gears).
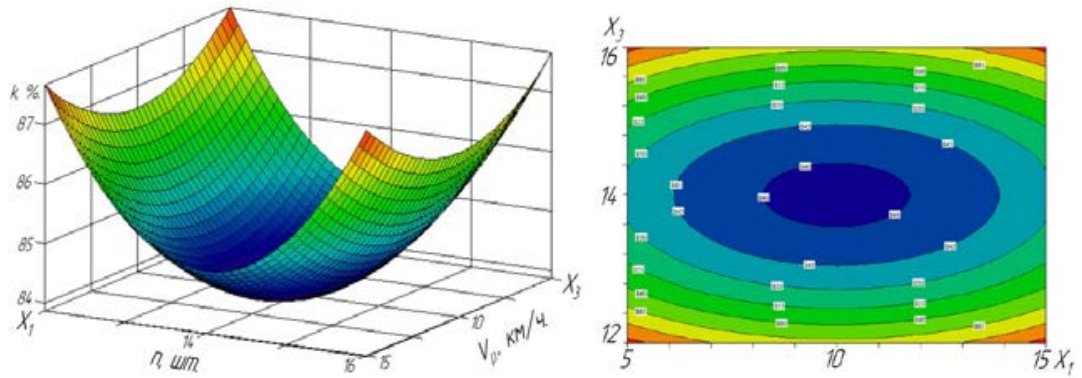

Fig. 2. The surface of the dependence of soil crumbling on the velocity of movement and the number of needles (gears) of the unit and its two-dimensional cross-section

Similarly, if the number of needles is optimal, the change in the response value is influenced by the disk diameter and speed of movement. To do this, it is necessary to substitute the optimal imaginary value of the number of needles in the regression equation $x_{3}=0,199$ (2). Then,

The equation takes the form:

$$
Y_{12}=84,91-1,851 \cdot x_{1}+2,523 \cdot x_{2}+1,2375 \cdot x_{1} \cdot x_{2}+1,038 \cdot x_{1}^{2}+2,768 \cdot x_{2}^{2}
$$

After the canonical transformation, the equation (9) with the angle of rotation $\alpha$ equal to $\left(-17,788^{\circ}\right)$, it will take the form (10), and the coefficients of this equation will be equal to $B_{11}=0,839, B_{22}=2,967$. Then the equation takes the form:

The surface shown in (Fig. 3) and obtained from the regression equation (10) is an elliptical paraboloid. The center of the surface is the minimum, since the coefficients of the equation are positive [4]. In this two-dimensional cross-section, the diameter of the working body affects the response value more significantly than the speed of movement, since the ellipse is expanded more along the axis corresponding to the speed of movement.
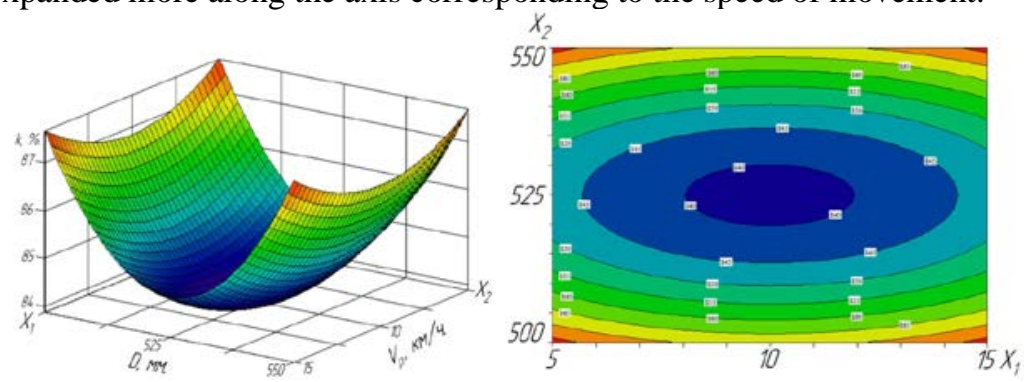

Fig. 3. The surface of the dependence of soil crumbling on the velocity and diameter of the disk and its two-dimensional cross-section 


\section{Conclusions}

On the basis of multifactor experiment planning, there were optimized the parameters and mode of operation of the proposed disk of the working body of rotary hoes: disk diameter is $518 \mathrm{~mm}$, the number of gears on a disk 14, a step arrangement of gears $0,118 \mathrm{~m}$, the number of gears that are simultaneously in the soil - three. The optimal working velocity of the hoe was $6.43 \mathrm{~km} / \mathrm{h}$.

\section{References}

1. Adler, Yu. P. Planning of the experiment under searching optimal conditions [Text] Yu. P. Adler, E. V. Markova, Yu. V. Granovsky. - M. : Nauka, 1976. - 278 p.

2. Serguntsov A. Harrowing of sowings with synchronous additional fertilizing / A. Serguntsov, N. Malashikhin // MATEC Web of Conferences 2018. - P. 05016.

3. Konovalov V. Constructive-technological diagram of the rotary-string cultivator and the definition of its main parameters / V. Konovalov, S. Konovalov, V. Igumnova // E3S Web of Conferences 126, 00039 (2019).

4. Experimental Studies of Parameters of Pneumatic Slot Sprayer / Evgeniy Ivanovich Trubilin, Svetlana Ivanovna Borisova, Vladimir Ivanovich Konovalov, Mikhail Ivanovich Chebotarev, Anatoliy Dmitrievich Gumbarov // International Journal of Emerging Trends in Engineering Research. - 2020. - Vol. 8, No. 1 January 2020, ISSN 2347-3983.

5. Unit for soil processing with fertilization / Patent for invention RUS 2629265. publ. 28.08.2017. Authors: Maslov G.G., Serguntsov A.S. 